\title{
Abordaje nutrigenómico de la obesidad: ¿dónde estamos?
}

\author{
A nutrigenomic approach to obesity: Where are we? \\ Abordagem nutrigenômica da obesidade: onde estamos?
}

\author{
Estefanía Zapata-Bravo', Rafael Adrián Pacheco-Orozco², César Payán-Gómez³, Julián López-Rippe²*
}

Recibido el: 29 de junio de 2020. Aceptado para publicación: 30 de agosto de 2020.

Publicado primero en línea: 7 de septiembre de 2020. https://doi.org/10.35454/rncm.v4n1.167

\section{Resumen}

La obesidad es una enfermedad multifactorial, es decir que resulta de la interacción de múltiples factores genéticos y ambientales. Para su estudio se hace necesario el uso de herramientas de investigación que permitan explorar mecanismos de interacción entre el genoma completo y la nutrición. La genómica nutricional que engloba la nutrigenética y la nutrigenómica ha estudiado el papel de los genes en la obesidad. Aunque estas dos últimas están íntimamente asociadas, toman un enfoque diferente para entender la relación entre los genes y la dieta. Se han encontrado diversas variantes genéticas asociadas a la susceptibilidad de la enfermedad, con el Índice de Masa Corporal, el porcentaje de grasa corporal, la circunferencia de la cintura y la relación cintura cadera, así como la interacción entre estas y el consumo de diferentes nutrientes como los hidratos de carbono y los lípidos. Se ha postulado que varias regiones del genoma están asociadas al control del peso corporal, y la forma como ciertos nutrientes pueden incluso modificar algunos procesos celulares que aumentan el riesgo de obesidad. Aún cuando estos hallazgos son de valioso significado, presentan limitaciones que impiden que hasta el momento tengan aplicación clínica.

El objetivo de esta revisión es describir los avances en la genómica nutricional respecto a la obesidad y cuál ha sido el papel y la aplicación de las ciencias ómicas en su estudio.

Palabras clave: nutrigenómica, genética, obesidad.

\section{Summary}

Obesity is a multifactorial disease resulting from the interaction of multiple genetic and environmental factors. Research tools that allow exploring mechanisms of interaction between the genome and nutrition are required for its study. Nutritional genomics encompassing nutrigenetics and nutrigenomics have studied the role of genes in obesity. Although the latter two are closely linked, they take a different approach to understanding the relationship between genes and diet. Several genetic variants associated with disease susceptibility, body mass index, body fat percentage, waist circumference and waist-to-hip ratio have been found, as well as interactions between these variants and intake of a variety of nutrients such as carbohydrates and lipids. Several genomic regions associated with body weight control and how certain nutrients may potentially modify cellular processes that increase the risk of obesity have been proposed. Although these findings are significant, they have limitations that currently prevent their clinical application.

The objective of this review is to describe the advances in nutritional genomics regarding obesity, and the role and use of omics technologies in its study.

Key words: Nutrigenomics; Genetics; Obesity.

\section{Resumo}

A obesidade é uma doença multifatorial, quer dizer, que resulta da interação de múltiplos fatores genéticos e ambientais. Para o seu estudo é necessário o uso de ferramentas de investigação que permitam explorar mecanismos de interação entre o genoma completo e a nutrição. A genômica nutricional que engloba a nutrigenética e a nutrigenômica tem estudado o papel dos genes na obesidade. Embora os dois estejam intimamente associados, eles adotam uma abordagem diferente para entender a relação entre genes e dieta. Se têm encontrado diversas variantes genéticas associadas à susceptibilidade da doença, como o Índice de Massa Corporal, a percentagem de gordura corporal, o perímetro abdominal e a relação cintura quadril, bem como a interação entre estes e o consumo de diferentes nutrientes como os carboidratos e lipídios. Postula-se que várias regiões do genoma estão associadas ao controle do peso corporal, e à forma como certos nutrientes podem até modificar alguns processos celulares que aumentam o risco de obesidade. Embora esses achados sejam de grande importância, eles apresentam limitações que impedem até ao momento, a sua aplicação clínica.

O objetivo desta revisão é descrever os avanços da genômica nutricional em relação à obesidade e qual tem sido o papel e a aplicação das ciências ômicas em seu estudo.

Palavras-chave: nutrigenômica, genética, obesidade. 
Escuela de Medicina y Ciencias de la Salud. Universidad del Rosario, Bogotá, D.C., Colombia.

2 Universidad El Bosque. Bogotá, D.C., Colombia.
3 Departamento de Biología. Facultad de Ciencias. Universidad del Rosario, Bogotá, D.C., Colombia.

*Correspondencia: Julian López-Rippe, MD

julianlopezrippe@gmail.com

\section{INTRODUCCIÓN}

Según la Organización Mundial de la Salud la obesidad es la acumulación anormal o excesiva de grasa que puede ser perjudicial para la salud. La forma más comúnmente utilizada para clasificar la obesidad es a través del Índice de Masa Corporal (IMC) en adultos y estándares de crecimiento en el caso de niños y adolescentes; los criterios para su diagnóstico se muestran en la Tabla 1. En 2016, 650 millones de adultos mayores de 18 años en el mundo tenían obesidad, tres veces la prevalencia que en $1975^{(1)}$. De continuar esta tendencia de crecimiento en la prevalencia de obesidad, para el año 2030 se espera que en el mundo aproximadamente $38 \%$ de los adultos tengan sobrepeso y $20 \% \operatorname{obesidad}^{(2)}$.

Tabla 1. Criterios de obesidad según la Organización Mundial de la Salud (OMS)

\section{Obesidad en adultos: \\ IMC mayor que $25 \mathrm{~kg} / \mathrm{m}^{2}=$ sobrepeso \\ IMC mayor o igual que $30 \mathrm{~kg} / \mathrm{m}^{2}=$ obesidad \\ IMC entre $30 \mathrm{~kg} / \mathrm{m}^{2}$ y $34,9 \mathrm{~kg} / \mathrm{m}^{2}$ = obesidad clase I \\ IMC entre $35 \mathrm{~kg} / \mathrm{m}^{2}$ y $39,9 \mathrm{~kg} / \mathrm{m}^{2}$ = obesidad clase II \\ IMC mayor o igual que $40 \mathrm{~kg} / \mathrm{m}^{2}$ = obesidad clase III}

Obesidad en niños menores de $\mathbf{5}$ años: peso para la altura mayor de 3 desviaciones estándar por encima de la mediana de los estándares de crecimiento infantil de la OMS.

Obesidad en niños de entre 5 y 19 años: mayor que 2 desviaciones estándar por encima de la mediana de referencia de crecimiento de la OMS.

IMC= Índice de Masa Corporal

Las tendencias de obesidad han demostrado que es una enfermedad prevalente en países desarrollados y en desarrollo. Estados Unidos es uno de los países que lidera la lista, con $42,4 \%$ de su población adulta mayor de 20 años con obesidad en $2017-2018^{(3)}$. En el caso de América Latina y el Caribe, la obesidad afecta a cerca de 140 millones de personas, observando una mayor prevalencia en países del Caribe como Bahamas
(36,2 \%), Barbados (31,3\%), Trinidad y Tobago $(31,1 \%)$ y Antigua y Barbuda $(30,9 \%)^{(4)}$. En México y Colombia, las estadísticas de sobrepeso y obesidad también muestran la importancia de esta problemática. En México, de acuerdo con la Encuesta Nacional de Salud y Nutrición (ENSANUT), para el año 2018 la prevalencia de sobrepeso y obesidad en menores de cinco años fue $6,1 \%$, en escolares de 33,2\%, en adolescentes de $33,6 \%$ y en los adultos $72,5 \%{ }^{(5)}$. En el caso de Colombia, para el año 2015 según la Encuesta Nacional de Situación Nutricional (ENSIN) 37,7 \% de la población joven y adulta presentaba sobrepeso, y 18,7\% tenía obesidad ${ }^{(6)}$.

La obesidad es considerada una enfermedad crónica y un factor de riesgo asociado al aumento de morbilidad y mortalidad por cáncer, enfermedades cardio-vasculares, diabetes, hipertensión arterial, enfermedades cerebrovasculares, apnea del sueño, discapacidad y osteoartritis. La obesidad se presenta cuando la ingesta calórica es mayor que el gasto calórico, resultando en un balance energético positivo que promueve la acumulación de triacilglicerol en el tejido adiposo. Dentro de la patogénesis de la obesidad están involucrados diversos factores, como estatus socioeconómico, cultural, y componentes genéticos y ambientales ${ }^{(7,8)}$. Estos últimos dos factores han cobrado gran importancia al momento de intentar explicar la razón por la cual no todos los individuos de una población se ven igualmente afectados. En 2016, se encontraron aproximadamente 140 genes de susceptibilidad a la obesidad que están asociados a herramientas como el IMC y de adiposidad (porcentaje de grasa corporal y circunferencia de la cintura); sin embargo, no explican la enfermedad en su totalidad. Por esto, es importante tener en cuenta la existencia de un ambiente promotor de obesidad que combina, dentro de sus factores más relevantes, los nutrientes dietarios, la edad, el género, la etnicidad, el tiempo de dormir, la cantidad de actividad física, el comportamiento sedentario, el estrés, el consumo de alcohol, cigarrillo, medicamentos y la depresión ${ }^{(9)}$. 
En 1990 empezó el Proyecto del Genoma Humano y en 2004 se liberó la primera versión de la secuenciación del genoma humano alcanzando un hito científico sin precedentes ${ }^{(10)}$. Gracias a los descubrimientos de este proyecto y a los avances en la nutrición se ha configurado una nueva disciplina, conocida como la genómica nutricional. Después de completar el mapeo del genoma humano se han realizado múltiples estudios de asociación para identificar los factores genéticos que pueden explicar la variabilidad de la respuesta metabólica a dietas específicas entre individuos ${ }^{(11)}$. La genómica nutricional busca un mejor tratamiento y prevención de las enfermedades por medio de una dieta individualizada ${ }^{(10)}$.

Se conoce que tanto la genética como el ambiente participan en el fenotipo de cada individuo, pero así mismo, factores ambientales modificables como la nutrición presentan un rol importante en el desarrollo de la obesidad y su progresión. Por lo anterior, la genómica nutricional, que engloba disciplinas como la nutrigenética y la nutrigenómica, ha estudiado el papel de los genes en la obesidad. Aunque estas dos áreas que trabaja la genómica nutricional están íntimamente asociadas, toman un enfoque diferente para entender la relación entre los genes y la dieta ${ }^{(12)}$. La nutrigenética estudia el efecto de las variaciones genéticas y su interacción con los nutrientes en el organismo ${ }^{(10)}$. Esto incluye la caracterización e identificación de variantes génicas asociadas o responsables de las respuestas diferenciales a los nutrientes. Su objetivo es generar recomendaciones personalizadas sobre el riesgo y beneficios de las dietas específicas o de componentes $\operatorname{dietarios}^{(13)}$. La nutrigenómica estudia la influencia de los nutrientes en la expresión génica e intenta rela- cionar los diferentes fenotipos resultantes con las respuestas celulares y genéticas (Figura 1). Se enfoca en el efecto de los nutrientes en el epigenoma, transcriptoma, proteoma y metaboloma. Esta disciplina puede llegar a tener implicaciones en la salud pública porque tiene el potencial de identificar personas con riesgo de desarrollar enfermedades metabólicas y orientar cambios en la dieta para prevenirlas ${ }^{(12)}$.

Esta revisión aborda en qué consiste la genómica nutricional, sus alcances, el uso de tecnologías del campo de las ciencias ómicas y su papel en el desarrollo y abordaje de la obesidad. Para lograr este objetivo se presenta una breve introducción a los conceptos básicos de la genética para entender más adelante la aplicación y el desarrollo de cada ciencia ómica y su aporte específico en el estudio de la obesidad desde la perspectiva tanto de la nutrigenética como de la nutrigenómica. Se realizó una búsqueda en la base de datos MEDLINE con los siguientes términos libres "nutrigenetics" OR "nutrigenomics" AND "obesity" sin límites en el periodo de publicación o el idioma.

La Tabla 2 presenta las definiciones de los términos básicos usados en esta revisión.

\section{LAS ÓMICAS EN EL ESTUDIO DE LA NUTRICIÓN Y LA OBESIDAD}

Al ser la obesidad una enfermedad multifactorial, es decir que resulta de la interacción de múltiples factores genéticos y ambientales, para su estudio se hace necesario el uso de herramientas de investigación que permitan explorar mecanismos de interacción entre el genoma completo y la nutrición. Para este fin, en los últimos años, se han empleado diferentes estrategias

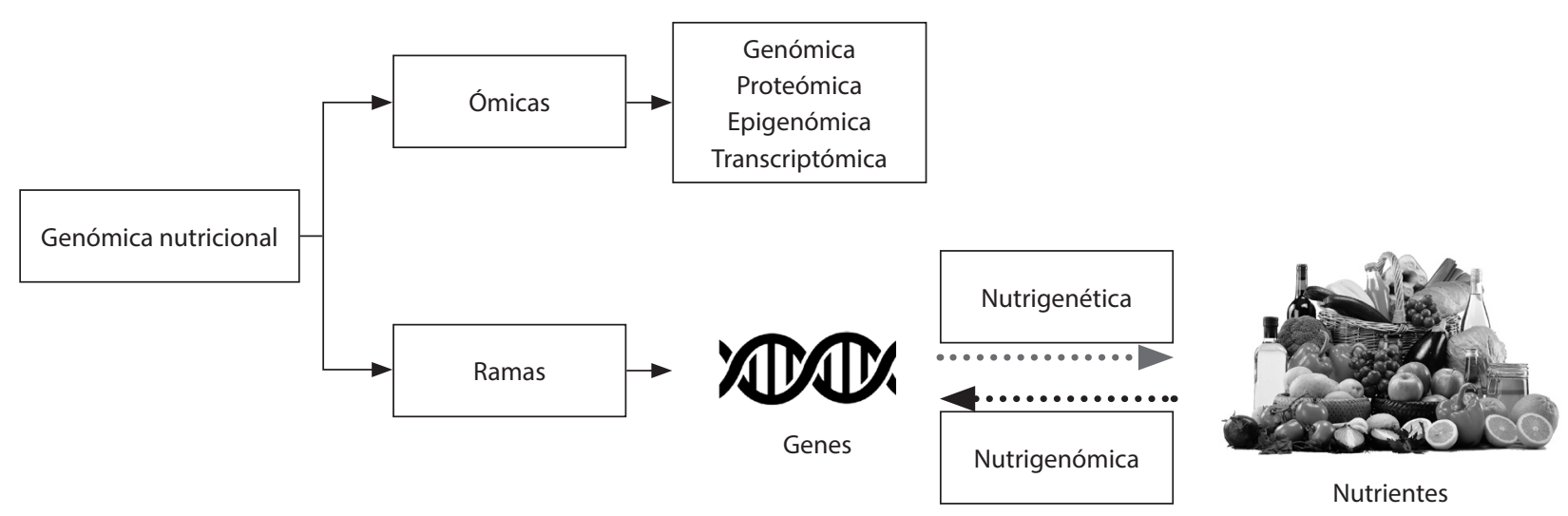

Figura 1. Genómica nutricional. Tomada y modificada de: Peña-Romero AC, et al. ${ }^{(13)}$. 
Tabla 2. Términos básicos usados en esta revisión ${ }^{(10,12)}$

\begin{tabular}{|c|c|}
\hline Término & Definición \\
\hline Heredabilidad & $\begin{array}{l}\text { Mide la proporción atribuible a diferencias genéticas de la varianza fenotípica en una } \\
\text { población. }\end{array}$ \\
\hline Ómicas & $\begin{array}{l}\text { La ómica es un sufijo que se utiliza para referirse al estudio del conjunto total de elementos } \\
\text { biológicos. Da origen a la denominación de la genómica, epigenómica y transcriptómica. }\end{array}$ \\
\hline Genómica nutricional & Estudia la interacción entre los genes, la dieta humana y su influencia en el metabolismo. \\
\hline Nutrigenómica & Efecto de la nutrición en los genes. \\
\hline Nutrigenética & Efecto de las variaciones genéticas en la interacción con los nutrientes. \\
\hline Genómica & Se refiere al estudio del genoma completo de un organismo. \\
\hline Epigenómica & $\begin{array}{l}\text { Estudio de las modificaciones o marcas a lo largo del genoma de un individuo que no } \\
\text { comprometen la secuencia propia de ADN. }\end{array}$ \\
\hline Transcriptómica & $\begin{array}{l}\text { Estudio del conjunto completo de transcripciones de ARN que produce el genoma, en } \\
\text { circunstancias específicas o en una célula específica. }\end{array}$ \\
\hline Nutrición de precisión & $\begin{array}{l}\text { Hace referencia a la nutrición personalizada con un enfoque más amplio en el que se } \\
\text { incluye la atención integral de la enfermedad, principalmente metabólica, en donde se } \\
\text { tiene en cuenta tanto al individuo como a su entorno. }\end{array}$ \\
\hline Loci & $\begin{array}{l}\text { Plural de “Locus”. Se refiere a la localización exacta de un gen o una secuencia de ADN } \\
\text { dentro del genoma. }\end{array}$ \\
\hline Variantes de nucleótido único (SNV) & Se refiere a un cambio en el ADN que compromete un solo par de bases. \\
\hline $\begin{array}{l}\text { Estudio de asociación del genoma } \\
\text { completo (GWAS, por sus siglas en } \\
\text { inglés) }\end{array}$ & $\begin{array}{l}\text { Estudios de asociación de genoma completo. Es una herramienta que permite el estudio de } \\
\text { múltiples marcadores genéticos a lo largo del genoma de muchas personas y su asociación } \\
\text { con la presencia de enfermedades. }\end{array}$ \\
\hline
\end{tabular}

ómicas que han sido determinantes para el desarrollo de la nutrigenómica y la nutrigenética ${ }^{(13-15)}$.

El adecuado funcionamiento de los organismos depende en gran medida de la información genética contenida en el ADN, el cual está compuesto por cuatro nucleótidos, Adenina (A), Guanina (G), Citosina (C) y Timina ( $\mathrm{T})$, unidos uno detrás de otro. El total del ADN de una especie se llama genoma, en el caso de los humanos el genoma está compuesto por 3.400 millones de nucleótidos. Todas las células con núcleo tienen en él una copia de la información genética del individuo. Algunas regiones del $\mathrm{ADN}$, los genes, pueden servir como molde para la síntesis de ARN, este proceso se conoce como transcripción, el cual es necesario para que la información genética se exprese y genere un impacto en el funcionamiento del organismo. El genoma humano contiene alrededor de 30.000 genes $^{(14)}$; sin embargo, no todos los genes se transcriben simultáneamente en una célula, ya que, dependiendo del tipo celular se expresa determinado conjunto de genes. Alrededor de $10 \%$ de los genes del genoma se transcriben en un momento específico en una célula ${ }^{(15)}$. Son estos genes los que determinan el comportamiento de las células en los tejidos. El conjunto de todos los transcritos presentes en un tejido se denomina transcriptoma ${ }^{(16)}$. El ARN sintetizado en el proceso de transcripción es usado por las células como fuente de información para la síntesis de proteínas.

El genoma de una persona tiene más de 80 millones de variaciones en su secuencia al compararlo con el de otras personas $^{(17)}$. Son estas variaciones en el ADN y su interacción con el medio ambiente las responsables de una parte de la individualidad de cada integrante de una especie. Estas variaciones pueden ser de ganancia o de pérdida de nucleótidos (inserción o deleción) o de cambio de un nucleótido por otro (sustitución). El estudio de todas las variaciones del genoma es parte del objeto de estudio de la genómica. Si alguna de estas variaciones se encuentra en un gen, el producto de la transcripción tendrá la variación, y por lo tanto, la proteína también tendrá una modificación en la secuencia, lo cual puede determinar modificaciones en la función 
de la proteína. El conocimiento de la secuencia del genoma ha hecho posible estudiar cómo el genoma y la dieta interactúan en la determinación de diferentes fenotipos. En el caso de la obesidad se han realizado diferentes investigaciones que evalúan la manera como la dieta y la presencia de variaciones en todo el genoma determinan el IMC, el riesgo de desarrollar obesidad y enfermedades metabólicas ${ }^{(10)}$.

La transcripción está regulada por diferentes mecanismos, uno de estos, es la epigenética que consiste en cambios en la estructura del $\mathrm{ADN}$ que hacen que un gen se exprese o no. Los cambios epigenéticos más conocidos son la metilación de las citocinas y las modificaciones en las histonas. Estas, son proteínas que interactúan con el $\mathrm{ADN}$ haciendo que esté o no compactado. El conjunto de todas las modificaciones epigenéticas en un genoma se llama epigenoma. Por lo tanto, la epigenómica investiga los mecanismos que alteran la expresión génica sin alterar la secuencia de $\mathrm{ADN}$. Estos incluyen la metilación de ADN, la modificación de histonas y los RNA no codificantes. El control epigenético de la expresión génica está involucrado en gran cantidad de procesos celulares y fisiológicos, además de ser susceptible a cambios por factores externos como la dieta, por lo que las interacciones entre los factores nutricionales y los cambios epigenéticos se han implicado en el desarrollo de la obesidad ${ }^{(18)}$.

\section{ÓMICAS EN EL ESTUDIO DE INTERACCIÓN GEN-AMBIENTE EN OBESIDAD}

El estudio de las interacciones gen-ambiente se realiza utilizando diferentes enfoques metodológicos. Los estudios caso-control, los estudios de cohorte prospectivos, ensayos aleatorizados de intervención y estudios en gemelos han sido algunos de estos enfoques ${ }^{(19)}$. Cada tipo de investigación tiene una metodología específica que hace que el conocimiento recolectado sea diferente. Los estudios caso-control pretenden comparar una exposición a factores genéticos y ambientales determinados en dos grupos poblaciones, el de los casos y los controles. Los casos prevalentes en una población son evaluados en un punto del tiempo, mientras que los controles son seleccionados de esa misma población. Por su parte, en los estudios de cohorte prospectivo los participantes son seguidos a través del tiempo, comparando la aparición del resultado entre los expuestos con los no expuestos, lo cual disminuye los sesgos de selección y de recolección. Finalmente, los ensayos clínicos son usualmente los más confiables por la aleatorización en las exposiciones; aunque, muchas veces no son viables en nutrigenómica por dificultades logísticas y económicas ${ }^{(19)}$.

\section{GENÓMICA Y OBESIDAD}

Los estudios de la genómica en la obesidad están enfocados en identificar variantes genéticas asociadas a la enfermedad, la respuesta al tratamiento o el pronóstico del paciente ${ }^{(14)}$. Los métodos genómicos sirven para detectar variaciones en el ADN, así como cambios en la estructura y organización cromosómica ${ }^{(20)}$. Las variantes de nucleótido único ( $\mathrm{SNV}$, por sus siglas en inglés) son el tipo de variantes genéticas más ampliamente estudiadas en el área de la nutrigenómica. Para el análisis de las SNV se usan dos enfoques principales, el estudio por genes candidatos y los de asociación de genoma completo (GWAS) ${ }^{(21)}$. El enfoque de genes candidatos incluye la selección de uno o varios genes que se cree pueden estar relacionados con el resultado de interés (hypothesis-driven), en los cuales se estudian las SNV por medio de reacción en cadena de la polimerasa o cada vez más, por secuenciación de nueva generación (NGS, por sus siglas en inglés). Por otro lado, el enfoque por GWAS estudia cientos de miles de SNV a lo largo del genoma, por lo que expande la búsqueda de asociaciones más allá de unos cuantos genes específicos; sin embargo, requiere un número grande de participantes en el estudio para tener suficiente poder estadístico. Se han realizado una gran cantidad de estudios de GWAS para identificar variantes de genes de obesidad que aumentan su susceptibilidad y se han descubierto con éxito múltiples loci de susceptibilidad y nuevas vías asociadas a las medidas de obesidad central ${ }^{(10,16)}$.

\section{ESTUDIOS DE VARIANTES GÉNICAS EN OBESIDAD}

En 2010 por medio de una expansión del estudio GIANT (Genetic Investigation of Antropometric Traits) que tenía como objetivo identificar loci genéticos para la susceptibilidad a la obesidad, examinaron las asociaciones entre el IMC y $\sim 2,8$ millones de SNV en hasta 123.865 individuos de ancestría europea, seguido de un análisis confirmatorio para 42 SNV en 125.931 individuos independientes. Lograron confirmar 14 loci de susceptibilidad a la obesidad, ya conocidos, e identificaron 18 nuevos loci asociados al IMC ${ }^{(20)}$. El consorcio del estudio, posteriormente, realizó un meta-análisis 
con estos datos en el que se determinó que varios de los nuevos loci apuntan a genes involucrados en vías hipotalámicas claves del equilibrio energético. Las variantes identificadas, en su mayoría, tienen un tamaño de efecto pequeño a muy pequeño, explicando solo $1 \%$ $-2 \%$ de la varianza del IMC ${ }^{(21)}$.

El estudio del efecto de las SNV en obesidad ha revelado interacciones con el consumo de micro y macronutrientes, alimentos específicos y respuesta a patrones dietarios ${ }^{(22)}$. El gen FTO (fat mass and obesityassociated) ha sido uno de los más estudiados en cuanto a riesgo de obesidad, y se ha encontrado que la presencia de las variantes rs9939609(A) y rs8050136(A) confieren mayor susceptibilidad a tener obesidad en el contexto de dietas altas en lípidos e hidratos de carbono, respectivamente. Estas variantes también se han encontrado asociadas a riesgo de diabetes mellitus tipo 2. Así mismo, variantes en el gen PPARG, involucrado en la regulación del metabolismo de la energía y con expresión principal en el tejido adiposo, se han visto asociadas a un mayor riesgo de obesidad ante la presencia de una dieta alta en lípidos. También se ha descrito una variante en el gen de la lactasa (LCT) que predispone a obesidad ante un consumo mayor a $8 \mathrm{~g} /$ día de productos lácteos $(1-2 \text { tazas de leche })^{(22)}$. Debido a la presencia de muchas variantes con efectos leves a moderados sobre la susceptibilidad a obesidad, se han desarrollado los puntajes de riesgo genético (GRS, por sus siglas en inglés), que examinan los efectos cumulativos de las SNV en interacciones dietarías y susceptibilidad a enfermedades ${ }^{(11)}$. Variantes en otros genes como los del receptor 4 de la melanocortina (MC4R) así como en los pertenecientes a la familia de apolipoproteínas (APOB, APOA1 y APOC3) que están relacionados con la regulación del consumo de alimentos y el metabolismo de los lípidos, respectivamente, han sido asociados a complicaciones de la obesidad como la diabetes mellitus tipo 2 y el síndrome metabólico ${ }^{(23-26)}$.

Otras de las áreas de estudio ha sido la relación SNV-dieta en la respuesta a intervenciones nutricionales dirigidas a la reducción de peso. Por ejemplo, ante la presencia de una dieta alta en proteína, la variante rs1558902(A) del gen FTO se encuentra asociada a una mayor pérdida de peso, mientras que la variante rs10830963(G) en el gen MTNR1B se asocia a menor pérdida de peso en mujeres. De la misma manera, la intervención nutricional con dietas altas en grasa ( $40 \%$ del total de la composición de macronutrientes) se asocia a menor pérdida de peso en pacientes con variantes en los genes TCF7L2 y PPM1K, en comparación con pacientes sin estas variantes ${ }^{(22)}$.

Después de los primeros estudios de GWAS para encontrar genes asociados a enfermedades complejas comunes, se descubrió que las asociaciones significativas que explican la heredabilidad eran muy pocas para casi todos los rasgos estudiados. La heredabilidad mide la proporción atribuible a diferencias genéticas de la varianza fenotípica en una población. Al compararse las estimaciones de heredabilidad esperada con los estudios tradicionales genéticos basados en gemelos, donde se ha demostrado que la herencia contribuye entre $40 \%$ y $75 \%$ de los casos de obesidad, la heredabilidad explicada fue siempre menor ${ }^{(16)}$. Aunque la heredabilidad del peso corporal es alta, solo una pequeña fracción de la varianza aún puede atribuirse a factores genéticos $\operatorname{conocidos}^{(13,14)}$.

Dado lo anterior, las variantes genéticas identificadas hasta ahora solo explican una pequeña proporción de la heredabilidad de las enfermedades, lo que sugiere que existe una heredabilidad faltante (missing heredability en inglés). Se puede llegar a pensar, que esas interacciones no identificadas de los genes pueden ser interacciones complejas con el ambiente, la dieta o los nutrientes $y$ ser un porcentaje de la heredabilidad faltante ${ }^{(27)}$.

\section{EPIGENÓMICA Y OBESIDAD}

La asociación entre los cambios epigenéticos y la obesidad fue observada clásicamente por medio de la "hipótesis de Barker", u origen fetal de las enfermedades, según la cual los fenotipos observados en los adultos tienen su origen durante la vida in utero, argumentando que los bebés que fueron concebidos en un ambiente de hambruna tienen mayor probabilidad de tener sobrepeso en la vida adulta ${ }^{(28)}$. Se ha comprobado que el perfil epigenético de estos pacientes en genes como LEP, IGF2 y ABCG1 se encuentra alterado, lo que sugiere que las marcas epigenéticas presentes al nacimiento pueden predecir el riesgo de obesidad en el futuro $^{(29)}$.

El método que se utiliza para hallar los cambios epigenéticos depende de si la intención es descubrir cambios desconocidos a lo largo del genoma o si se busca evaluar el estado de metilación en regiones específicas de interés. Los métodos más conocidos incluyen el uso de bisulfito, el cual es capaz de diferenciar las bases nitrogenadas metiladas de las no metiladas. Como también la inmunoprecipitación de cromatina (ChIP), que 
utiliza anticuerpos específicos para purificar e identificar los sitios metilados ${ }^{(23)}$.

Se conocen aproximadamente 187 regiones del genoma cuyos cambios epigenéticos están asociadas al peso corporal. La mayoría de estas regiones se encuentran cerca de genes relacionados con la homeostasis del tejido adiposo y la resistencia a la insulina ${ }^{(30)}$. A través del uso de Estudios de Asociación de Epigenoma Completo (EWAS, por su siglas en inglés) tanto en sangre periférica como en tejido adiposo, se han encontrado asociaciones significativas entre el IMC y el estado de metilación de genes como HIF3A, CPT1A, ABCG1, y SREBF1, estas alteraciones son causadas directamente por los niveles de adiposidad, y no al revés ${ }^{(19,20,31)}$.

En modelos animales también se ha encontrado que la suplementación con una dieta alta en grasas (700 $\mathrm{mg} / \mathrm{kg}$ o $60 \%$ del total de macronutrientes) y sucrosa, lleva a un aumento en la metilación del gen LEP, así como a una disminución en la metilación de genes como OPRM1, PENK y DAT, todos estos genes de neuropéptidos involucrados en el control del consumo de alimentos, lo cual lleva a un aumento del riesgo de obesidad. Reconociendo el carácter reversible de las marcas epigenéticas, se han estudiado intervenciones nutricionales específicas dirigidas a cambiar estas alteraciones con la intención de prevenir el desarrollo de enfermedades crónicas. Se ha visto que los polifenoles de las manzanas, así como el pteroestilbeno, influyen en los patrones de metilación de ciertos genes asociados al metabolismo de lípidos y contribuyen en la prevención del desarrollo de obesidad ${ }^{(22)}$.

\section{TRANSCRIPTÓMICA Y OBESIDAD}

Para extender la comprensión de cómo el genoma interactúa con la dieta en la obesidad, se ha investigado también cómo se comporta el transcriptoma en personas con obesidad en diferentes diseños experimentales. Se ha comparado a personas con obesidad con personas con peso normal, para estudiar el cambio en la expresión de los genes después de intervenciones para la pérdida de peso como la cirugía bariátrica, o para determinar el impacto en la expresión de ciertos genes de dietas específicas. Como cada tejido tiene un transcriptoma específico este tipo de estudios tienen la limitación de que dependiendo del tejido que se analice los resultados pueden ser diferentes. La mayoría de los investigadores se han decantado por el uso de células mononucleares de sangre periférica. Las razones de esto es la facilidad de acceso, la no invasividad en la toma y que se propone que puede reflejar estados fisiológicos de órganos internos como el tejido adiposo y el hígado ${ }^{(32,33)}$.

La comparación del transcriptoma de personas con obesidad con personas normales ha permitido identificar potenciales biomarcadores para el desarrollo de obesidad y de complicaciones asociadas a la misma. Jang y colaboradores ${ }^{(34)}$ estudiaron los transcriptomas de células mononucleares de sangre periférica de 30 personas con obesidad y 20 personas sin obesidad, encontraron que la expresión de 9 genes se relacionaba significativamente con índices de obesidad. Por ejemplo, la expresión de TFEC y CCL2, dos genes involucrados en la regulación de la respuesta inmune, estaba negativamente correlacionada con el IMC, la cantidad de grasa corporal, el índice cintura-cadera y las concentraciones de leptina. Adicionalmente, Sánchez y colaboradores $^{(35)}$ al estudiar 15 niños con obesidad y compararlos con 15 con peso normal, encontraron que genes relacionados con fosforilación oxidativa mitocondrial estaban consistentemente menos expresados en los niños con obesidad. Algo similar fue identificado en adultos con diabetes tipo 2, lo que sugiere que la disminución en la expresión de estos genes puede ser un marcador temprano de futuras alteraciones metabólicas en pacientes con obesidad ${ }^{(36)}$. La principal limitación de este tipo de investigaciones es el pequeño número de personas estudiadas, lo que hace que se describan muy bien las características de los pacientes en el estudio, pero dificulta la generalización de los hallazgos.

Una forma de aumentar el poder estadístico de los estudios individuales es combinarlos por medio de un meta-análisis. Ortiz-Dosal y colaboradores ${ }^{(37)}$ combinaron el resultado de 21 investigaciones donde compararon los niveles de expresión de microRNA (miRNA), un tipo de RNA involucrado en la regulación de RNA mensajero (RNAm). En total encontraron 22 miRNA sobre-expresados y 9 miRNA reprimidos en personas con obesidad comparadas con personas delgadas. Las vías en las cuales están involucrados estos miRNAs son la PI3k/Akt y el metabolismo de ácidos grasos. Los autores proponen que la desregulación de PI3k/Akt podría explicar en parte el mayor riesgo de diabetes tipo 2 en personas con obesidad. Además de estar asociada también al desarrollo de algunos tipos de cáncer.

Con una aproximación similar a la de Ortiz y colaboradores $^{(37)}$, Langi y colaboradores ${ }^{(38)}$ combinaron 17 estudios en los que se identificaron cambios en los niveles de expresión de miRNA después de la realización de cirugía bariátrica. Ellos encontraron que la expre- 
sión de 14 miRNA se modificó después de la cirugía. Las vías celulares reguladas por estos miRNA incluyen cáncer, metabolismo de ácidos grasos, vías de señalización y enfermedades infecciosas. Estas vías podrían estar relacionadas con los mecanismos moleculares responsables de la pérdida de peso y con la remisión de las comorbilidades después de la cirugía bariátrica.

Estos hallazgos demuestran el potencial del estudio del transcriptoma para entender los procesos celulares y moleculares causales de la obesidad y sus complicaciones. Sin embargo, los tamaños de muestra limitados y la heterogeneidad de los diseños experimentales y de las técnicas usadas hacen necesario que se realicen estudios adicionales con mayor número de pacientes de diferentes poblaciones, además de validación en el laboratorio para que los resultados puedan ser generalizados.

\section{RECOMENDACIONES Y CONCLUSIONES}

Hasta la fecha los avances en materia de genómica nutricional han demostrado tener su aplicación en diferentes instancias puntuales en lo que respecta a la salud, tal y como se ha mostrado ya a través del estudio de las ómicas. La transición epidemiológica ha generado que se usen diferentes aproximaciones para el estudio de las enfermedades crónicas. Dentro de estas nuevas formas de abordar las enfermedades crónicas, en especial las de interés público, la nutrigenómica ha surgido como una herramienta de estudio. En la actualidad, la nutrigenética presenta una aplicabilidad clínica más fácilmente identificable como es el caso de aquellas patologías agudas hereditarias como la galactosemia y la fenilcetonuria. Sin embargo, el área de estudio de la nutrigenómica ha presentado mayor interés dentro de los investigadores, ya que las enfermedades crónicas como el síndrome metabólico, diabetes tipo 2, la obesidad y las enfermedades cardiovasculares han demostrado ser prevenibles por medio de un estilo de vida saludable, dentro del cual la dieta es un factor clave junto a las medidas sanitarias ${ }^{(10)}$.

En materia de obesidad, en lo que respecta al campo de la genómica nutricional, se han visto hallazgos importantes sobre las diferentes variantes asociadas tanto a aumento del IMC y perímetro abdominal, como a susceptibilidades a diabetes mellitus tipo 2 y síndrome metabólico, así como una interacción entre estas y el consumo de diferentes nutrientes como los hidratos de carbono y los lípidos. Sin embargo, estas asociaciones a pesar de ser significativas muy pocas realmente explican el fenotipo complejo de la obesidad y su heredabilidad. Desde una perspectiva epigenética se han involucrado varias regiones asociadas al control del peso corporal, y cómo ciertos nutrientes pueden incluso modificar procesos celulares como la metilación aumentando el riesgo de obesidad. Así mismo, diferentes biomarcadores se han identificado, tanto en población adulta como pediátrica, que se relacionan de manera tanto inversa como directa. Aún cuando estos hallazgos son de valioso significado, presentan limitaciones porque no permiten explicar en su totalidad la problemática o metodológicamente no podrían ser extrapolables, lo que impide que hasta el momento tengan una aplicación clínica relevante.

La nutrigenómica tiene el potencial de identificar factores predictores genéticos que responden a la dieta y son relevantes para la enfermedad, por lo que presenta un atractivo dado su potencial y aplicabilidad en el contexto de la nutrición personalizada ${ }^{(15)}$. La nutrición de precisión toma en cuenta la variabilidad individual, el genoma, el metaboloma, el microbioma y otras composiciones para diseñar intervenciones ${ }^{(16)}$. Uno de sus objetivos es diseñar recomendaciones nutricionales más completas y dinámicas basadas en parámetros cambiantes e interactivos para tratar o prevenir los trastornos metabólicos. Incluye hábitos alimentarios, comportamiento alimentario, actividad física, la microbiota y el metaboloma. Aunque numerosos genes y polimorfismos ya se han identificado como factores relevantes en esta respuesta heterogénea a la ingesta de nutrientes, la evidencia clínica que respalda estas relaciones es insuficiente para establecer un marco integral para intervenciones nutricionales personalizadas en la mayoría de los $\operatorname{casos}^{(10)}$.

\section{Financiación}

El presente artículo no tuvo financiación

\section{Declaración de conflicto de interés}

Los autores declaran no tener conflicto de interés

\section{Declaración de autoría}

E.Z, R.P, C.P y J.L realizaron la búsqueda de la evidencia, escribieron, revisaron el artículo y validaron su versión final.

\section{Referencias bibliográficas}

1. World Health Organization. Obesity and Overweight [Internet]. 1 April 2020. [Fecha de consulta: 21 de mayo de 
2020]. Disponible en: https://www.who.int/news-room/ fact-sheets/detail/obesity-and-overweight.

2. Hruby A, Hu FB. The Epidemiology of Obesity: A Big Picture. Pharmacoeconomics. 2015; 33(7): 673-89. doi: https://doi. org/10.1007/s40273-014-0243-x.

3. Hales CM, Carroll MD, Fryar CD, Ogden CL. Prevalence of Obesity and Severe Obesity Among Adults: United States, 2017-2018 [Internet]. Centers for Disease Control and Prevention (CDC); February 2020. [Fecha de consulta: 21 de mayo de 2020]. Disponible en: https://www.cdc.gov/nchs/ products/databriefs/db360.htm.

4. Organización Panamericana de la Salud, Organización Mundial de la Salud. Sobrepeso Afecta a Casi La Mitad De La Población De Todos Los Países De América Latina y El Caribe Salvo Por Haití [Internet]. Santiago de Chile; 19 de enero de 2017. [Fecha de consulta: 21 de mayo de 2020]. Disponible en: https://www.paho.org/col/index.php?option=com_con tent\&view=article \&id=2686: sobrepeso-afecta-a-casi-lamitad-de-la-poblacion-de-todos-los-paises-de-america-latinay-el-caribe-salvo-por-haiti\&Itemid= $=562$.

5. Shamah-Levy T, Campos-Nonato I, Cuevas-Nasu L, Hernández-Barrera L, Morales-Ruán MDC, RiveraDommarco J, et al. Overweight and obesity in Mexican vulnerable population. Results of Ensanut 100k. Salud Pública Méx. 2019; 61(6): 852-65. doi: https://doi.org/10.21149/10585.

6. Instituto Colombiano de Bienestar Familiar. ENSIN: Encuesta Nacional De Situación Nutricional 2015. [Fecha de consulta: 21 de mayo de 2020]. Disponible en: https://www. icbf.gov.co/bienestar/nutricion/encuesta-nacional-situacionnutricional.

7. Ritchie H, Roser M. Obesity [Internet]. Our world in data. 2017. [Fecha de consulta: 21 de mayo de 2020]. Disponible en: ourworldindata.org/obesity.

8. Smith KB, Smith MS. Obesity Statistics. Prim Care. 2016;43(1):121-35. doi: https://doi.org/10.1016/j. pop.2015.10.001.

9. Castillo JJ, Orlando RA, Garver WS. Gene-Nutrient Interactions and Susceptibility to Human Obesity. Genes Nutr. 2017;12:29. doi: https://doi.org/10.1186/s12263017-0581-3.

10. Mutch DM, Wahli W, Williams fecon G. Nutrigenomics and nutrigenetics: the emerging faces of nutrition. FASEB J. 2005;19(12):1602-16. doi: https://doi.org/10.1096/fj.053911 rev.

11. Phillips CM. Nutrigenetics and Metabolic Disease: Current Status and Implications for Personalised Nutrition. Nutrients. 2013; 5(1):32-57. doi: https://doi.org/10.3390/nu5010032.

12. de Toro-Martín J, Arsenault BJ, Després J-P, Vohl M-C. Precision Nutrition: A Review of Personalized Nutritional Approaches for the Prevention and Management of Metabolic Syndrome. Nutrients. 2017; 9(8): 913. doi: https://doi. org/10.3390/nu9080913.

13. Peña-Romero AC, Navas-Carrillo D, Marín F, Orenes-Piñero E. The future of nutrition: Nutrigenomics and nutrigenetics in obesity and cardiovascular diseases. Crit Rev Food Sci Nutr. 2018;58(17):3030-41. doi: https://doi.org/10.1080/10408 398.2017.1349731.

14. Pertea M, Shumate A, Pertea G, Varabyou A, Breitwieser FP, Chang Y-C, et al. CHESS: a new human gene catalog curated from thousands of large-scale RNA sequencing experiments reveals extensive transcriptional noise. Genome Biol. 2018; 19(1): 208. doi: https://doi.org/10.1186/s13059-018-1590-2.

15. García-Ortega LF, Martínez O. How Many Genes Are Expressed in a Transcriptome? Estimation and Results for RNA-Seq. PloS one. 2015; 10(6): e0130262. doi: https:// doi.org/10.1371/journal.pone.0130262.

16. Olivier M, Asmis R, Hawkins GA, Howard TD, Cox LA. The Need for Multi-Omics Biomarker Signatures in Precision Medicine. Int J Mol Sci. 2019; 20(19):4781. doi: https://doi. org/10.3390/ijms20194781.

17. 1000 Genomes Project Consortium, Auton A, Brooks LD, Durbin RM, Garrison EP, Kang HM, et al. A global reference for human genetic variation. Nature. 2015; 526(7571):68-74. doi: https://doi.org/10.1038/nature15393.

18. Pigeyre M, Yazdi FT, Kaur Y, Meyre D. Recent progress in genetics, epigenetics and metagenomics unveils the pathophysiology of human obesity. Clin Sci. 2016; 130(12): 94386. doi: https://doi.org/10.1042/CS20160136.

19. Huang T, Hu FB. Gene-environment interactions and obesity: recent developments and future directions. BMC Med Genomics. 2015; 8(Suppl 1): S2. doi: https://doi. org/10.1186/1755-8794-8-S1-S2.

20. Speliotes EK, Willer CJ, Berndt SI, Monda KL, Thorleifsson G, Jackson AU, et al. Association analyses of 249,796 individuals reveal eighteen new loci associated with body mass index. Nat Genet. 2010;42(11):937-48. doi: https://doi. org/10.1038/ng.686.

21. Hebebrand J, Volckmar A-L, Knoll N, Hinney A. Chipping Away the 'Missing Heritability': GIANT Steps Forward in the Molecular Elucidation of Obesity - but Still Lots to Go. Obes Facts. 2010;3(5):294-303. doi: https://doi. org/10.1159/000321537.

22. Ramos-Lopez O, Milagro FI, Allayee H, Chmurzynska A, Choi MS, Curi R, et al. Guide for Current Nutrigenetic, Nutrigenomic, and Nutriepigenetic Approaches for Precision Nutrition Involving the Prevention and Management of Chronic Diseases Associated with Obesity. J Nutrigenet Nutrigenomics. 2017;10(1-2):43-62. doi: https://doi. org/10.1159/000477729.

23. Mousavizadeh Z, Hosseini-Esfahani F, Javadi A, Daneshpour MS, Akbarzadeh M, Mirmrian P, et al. The interaction between dietary patterns and melanocortin-4 receptor polymorphisms in relation to obesity phenotypes. Obes Res Clin Pract. 2020;14(3):249-56. doi: https://doi.org/10.1016/j. orcp.2020.04.002

24. Vázquez-Moreno $\mathrm{M}$, Zeng $\mathrm{H}$, Locia-Morales $\mathrm{D}$, PeraltaRomero J, Asif H, Maharaj A, et al. The Melanocortin 4 Receptor p.Ile269Asn Mutation Is Associated with Childhood 
and Adult Obesity in Mexicans. J Clin Endocrinol Metab. 2020;105(4): dgz276. doi: https://doi.org/10.1210/clinem/ dgz276.

25. Su X, Peng D. The exchangeable apolipoproteins in lipid metabolism and obesity. Clin Chim Acta. 2020;503:128-35. doi: https://doi.org/10.1016/j.cca.2020.01.015.

26. Kumar A, Shalimar, Walia GK, Gupta V, Sachdeva MP. Genetics of nonalcoholic fatty liver disease in Asian populations. J Genet. 2019;98:29.

27. Heianza Y, Qi L. Gene-Diet Interaction and Precision Nutrition in Obesity. Int J Mol Sci. 2017;18(4):787. doi: https://doi.org/10.3390/ijms18040787.

28. Almond D, Currie J. Killing Me Softly: The Fetal Origins Hypothesis. J Econ Perspect. 2011; 25(3):153-72. doi: https://doi.org/10.1257/jep.25.3.153.

29. Burdge GC, Hoile SP, Lillycrop KA. Epigenetics: are there implications for personalised nutrition? Curr Opin Clin Nutr Metab Care. 2012; 15(5):442-7. doi: https://doi. org/10.1097/MCO.0b013e3283567dd2.

30. McCaffery J. Precision behavioral medicine: Implications of genetic and genomic discoveries for behavioral weight loss treatment. Am Psychol. 2018; 73(8):1045-55. doi: https:// doi.org/10.1037/amp0000253.

31. Wahl S, Drong A, Lehne B, Loh M, Scott WR, Kunze S, et al. Epigenome-wide association study of body mass index, and the adverse outcomes of adiposity. Nature. 2017; 541(7635):81-6. doi: https://doi.org/10.1038/nature20784.

32. Oliver P, Reynés B, Caimari A, Palou A. Peripheral blood mononuclear cells: a potential source of homeostatic imbalance markers associated with obesity development. Pflugers
Arch. 2013;465(4):459-68. doi: https://doi.org/10.1007/ s00424-013-1246-8.

33. de Mello FVD, Kolehmanien M, Schwab U, Pulkkinen L, Uusitupa M. Gene expression of peripheral blood mononuclear cells as a tool in dietary intervention studies: What do we know so far? Mol Nutr Food Res. 2012; 56(7):1160-72. doi: https://doi.org/10.1002/mnfr.201100685.

34. Jang K, Tong T, Lee J, Park T, Lee H. Altered Gene Expression Profiles in Peripheral Blood Mononuclear Cells in Obese Subjects. Obes Facts. 2020;13(3): 375-85. doi: https://doi. org/10.1159/000507817.

35. Sanchez J, Pico C, Ahrens W, Foraita R, Fraterman A, Moreno LA, et al. Transcriptome analysis in blood cells from children reveals potential early biomarkers of metabolic alterations. Int J Obes. 2017; 41(10):1481-8. doi : https://doi.org/10.1038/ ijo.2017.132.

36. Takamura T, Honda M, Sakai Y, Ando H, Shimizu A, Ota T, et al. Gene expression profiles in peripheral blood mononuclear cells reflect the pathophysiology of type 2 diabetes. Biochem Biophys Res Commun. 2007; 361(2): 379-84. doi: https:// doi.org/10.1016/j.bbrc.2007.07.006.

37. Ortiz-Dosal A, Rodil-Garcia P, Salazar-Olivo LA. Circulating microRNAs in human obesity: a systematic review. Biomarkers. 2019; 24(6): 499-509. doi: https://doi.org/10. 1080/1354750X.2019.1606279.

38. Langi G, Szczerbinski L, Kretowski A. Meta-Analysis of Differential miRNA Expression after Bariatric Surgery. J Clin Med. 2019;8(8):1220. doi: https://doi.org/10.3390/ jcm8081220. 